\title{
REVIEW
}

\section{Prediction of coronary vessel involvement on the basis of atherosclerosis risk factor analysis}

\author{
Kovarnik $\mathrm{T}^{1}$, Kral A ${ }^{1}$, Skalicka $\mathrm{H}^{1}$, Skalicka L ${ }^{1}$, Dostal $\mathrm{O}^{1}$, Kralik L ${ }^{2}$, Martasek $\mathrm{P}^{2}$, Aschermann $\mathrm{M}^{1}$, \\ Horak $\mathrm{J}^{1}$, Linhart $\mathrm{A}^{1}$, Wahle $\mathrm{A}^{3}$, Sonka $\mathrm{M}^{3}$ \\ Second Department of Internal Medicine, Cardiology and Angiology, General University Hospital, First Faculty of Medicine, \\ Charles University, Prague, Czech Republic. tkovarnik@yahoo.com
}

\begin{abstract}
The prediction of coronary vessel involvement by means of noninvasive tests is one of the fundamental objectives of preventive cardiology. This review describes the current possibilities of coronary vessel involvement prediction by means of ultrasonographic examination of carotid arteries, analysis of polymorphisms in the genes encoding enzymes responsible for production of nitric oxide and carbon monoxide and assessment of levels of certain proinflammatory cytokines. In the presented work these noninvasive markers are correlated with the extent of coronary vessel involvement as assessed by coronary angiography, intravascular ultrasound and virtual histology (Fig. 5, Ref. 40). Full Text in PDF www.elis.sk.

Key words: coronary artery disease, atherosclerosis, intravascular ultrasound, gene polymorphism, proinflammatory cytokines, intima-media thickness.
\end{abstract}

Coronary artery disease (CAD) is the most frequent cause of death in the developed countries. In the USA, CAD is diagnosed in $12 \%$ of the population, leading to approximately 4 million hospital admissions per year (1). More than $50 \%$ of acute coronary syndrome (ACS) cases occur in patients without any previous symptoms because only $20 \%$ of acute coronary vessel occlusions are caused by stenoses of over $75 \%$ (2). This is one of the fundamental subjects of preventive cardiology, since ACS leads to substantial personal and social consequences, including sudden death and chronic heart failure with the need for lifetime pharmacologic and possibly nonpharmacologic treatments (cardiac resynchronization therapy, implantable cardioverter-defibrillators), which have a negative impact on work and social assertion.

Therefore, in recent years, much attention has been focused on the development of new methods capable of detecting asymptomatic patients at high risk for ACS before it occurs. The pathophysiology of ACS is currently understood as an interplay of factors that include an unstable patient (accumulation of risk factors), high-risk blood (prothrombogenic state) and a high-risk plaque. The following types of plaques are considered unstable: eccentric

\footnotetext{
${ }^{1}$ Second Department of Internal Medicine, Cardiology and Angiology, General University Hospital, First Faculty of Medicine, Charles University, Prague, Czech Republic, ${ }^{2}$ Department of Pediatrics, General University Hospital, First Faculty of Medicine, Charles University, Prague, Czech Republic, and ${ }^{3}$ Dept of Electrical and Computer Engineering, The University of Iowa, Iowa City IA. USA

Address for correspondence: T. Kovarnik, MD, Second Department of Internal Medicine, Cardiology and Angiology, General University Hospital, U Nemocnice 2, CZ-128 00 Prague 2, Czech Republic.

Phone: +420.2.24962605, Fax: +420.2.24912154

Acknowledgement: This work was supported by IGA (Internal Grant Agency of Czech Ministry of Health) NR 9214-3 grant and by National Institutes of Health (U.S.A.), grant R01 HL063373.
}

plaques, bulky plaques compensated for by positive remodeling (an adaptive reaction of the artery to plaque progression which maintains sufficient vessel lumen) $(3,4)$ and ruptured plaques with superimposed intracoronary thrombosis (5).

\section{Possibilities diagnosing high-risk coronary vessel involvement}

The gold standard for coronary vessel evaluation is the coronary angiography (CAG). However, this technique does not visualize the atherosclerotic plaque per se. This visualization is possible with the use of intravascular ultrasound (IVUS). During this examination, a probe that allows for the visualization of the vessel wall and the atherosclerotic plaque is inserted into the vessel lumen (Fig. 1), followed by the subsequent evaluation of the above-mentioned high-risk morphological features (6) (Fig 2, 3). An additional risk factor for the development of ACS is plaque composition. Plaques leading to ACS contain a higher amount of lipid and necrotic matter (7). In vivo, plaque composition can be evaluated by means of virtual histology $(\mathrm{VH})$, which correlates well with actual histology (8). Virtual histology is an imaging technique that is based on a different way of analyzing the signal acquired during the IVUS examination. In contrast to IVUS examination, which assesses the amplitude of the ultrasound signal, VH assesses the frequency of the reflected ultrasound signal, which is specific for different plaque components (calcification, fibrous and fibrolipid tissue, necrotic core) (Figs 4, 5). A newly recognized type of plaque is the so-called thin-cap fibroatheroma (TCFA). Since one of the criteria for TCFA is the width of the fibrous cap of less than $65 \mu \mathrm{m}$, a value below the resolution capacity of the IVUS, TCFA is classified as either TCFA (a pathological-anatomical description of the lesion) or VH-TCFA (TCFA diagnosed by virtual histology). The criteria for VH-TCFA is a plaque comprised of more than $10 \%$ necrotic tissue in at least three consecutive cross- 


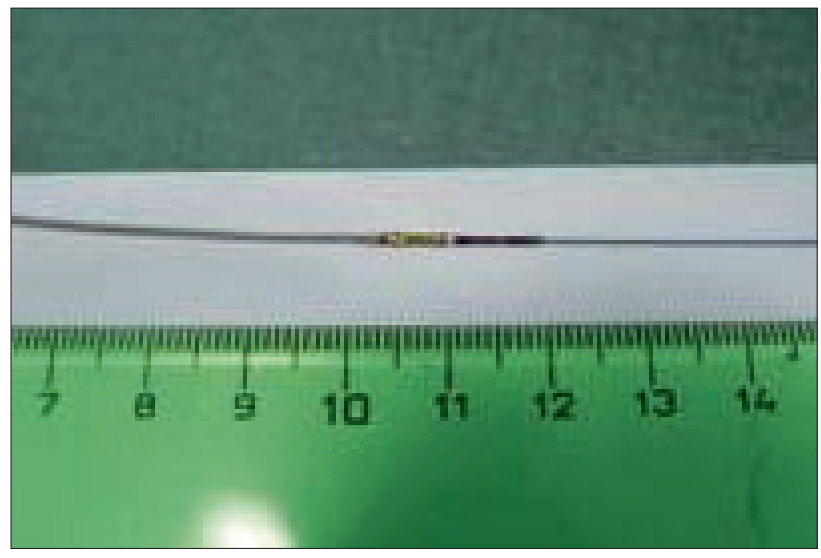

Fig. 1. IVUS probe on an intracoronary guidewire.

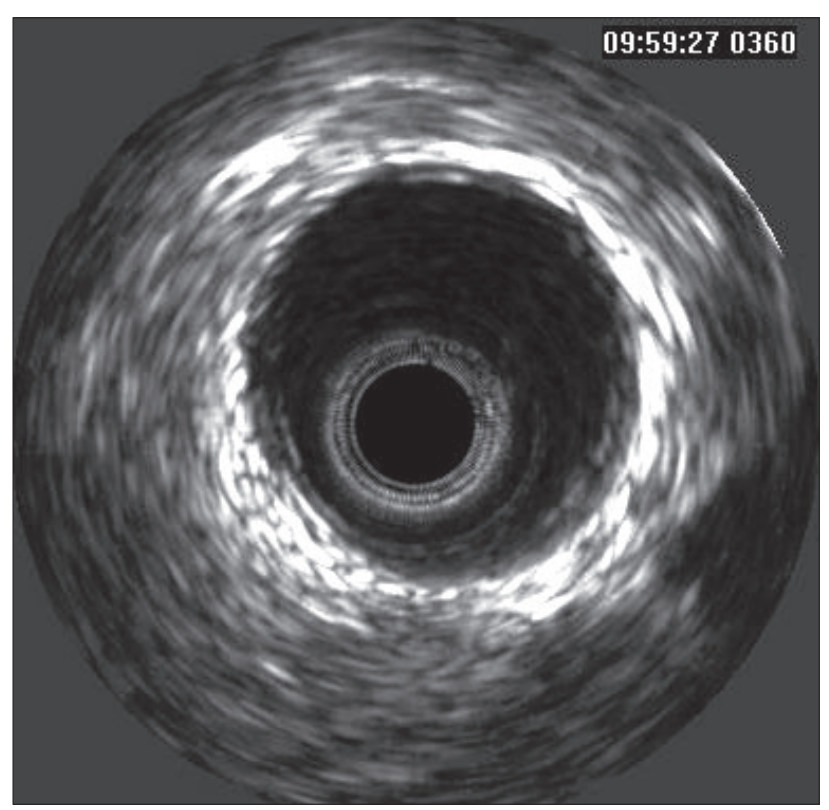

Fig. 2. IVUS picture of a normal coronary artery. sections and direct contact of the necrotic core with the vessel lumen (since the thin fibrous cap cannot be directly visualized) (9).

It has been established that during the gradual process of plaque enlargement, the absolute amounts of fibrous and lipid components of the plaque increase. That means that calcification and especially necrotic tissue are related to the development of an unstable plaque (9). Marso et al published an interesting study this year in which they evaluated the relationship between the type of coronary vessel involvement and the Framingham risk score. The type of coronary vessel involvement was assessed by IVUS and VH. The data from 531 patients included in a worldwide prospective VH-IVUS registry from 37 participating centers were evaluated in this study. Positive correlations were observed between the Framingham score and the atherosclerotic plaque volume and the presence of VH-TCFA. This finding corresponds well with the results from previous studies (1).

In the PROSPECT trial, including 697 patients who underwent a very detailed assessment of all three main coronary vessels, including IVUS and VH, in the acute phase of ACS were prospectively followed for three years. The goal of the study was to ascertain whether there are certain plaque characteristics that allow for the discrimination between plaques that will subsequently lead to the development of coronary syndromes and those that are not dangerous to their carrier. In a multivariate analysis, it was established that lesions leading to the development of coronary syndromes during the follow-up were bulkier (plaque burden $>70 \%$; this parameter expresses the plaque percentual proportion at a certain vessel point), were more frequently the VH-TCFA type of plaque and had a lumen smaller than $4 \mathrm{~mm}^{2}(2,10)$.

\section{The use of ultrasonographic examination of the carotid arter- ies in the prediction of coronary involvement}

Ultrasonographic examination of the carotid arteries is a noninvasive method facilitating the measurement of vessel lumen size and the intima and media thickness (IMT) and the assessment of the presence and extent of atherosclerotic plaques. In the context of predicting coronary vessel involvement, it must be emphasized

Plaque area

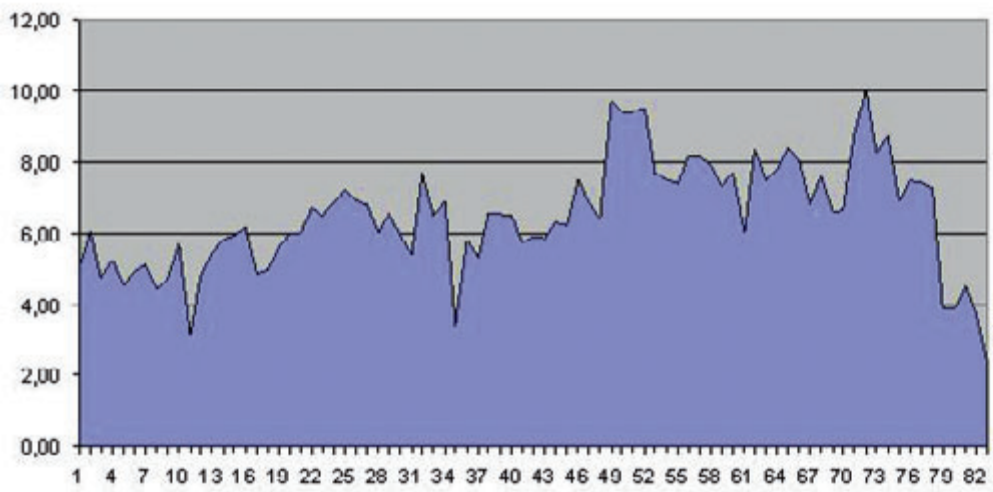

Fig. 3. Evaluation of plaque volume in an examined segment of a coronary artery. 
that atherosclerotic involvement of the carotid arteries is a strong predictor of coronary vessel involvement. The IMT value in the area of the common carotid artery correlates with the severity of coronary vessel involvement (11). For the IMT values greater than $1.15 \mathrm{~mm}$, there is a $94 \%$ probability of the presence of CAD. Only $1.9 \%$ of patients with triple vessel disease according to CAG have an IMT value of less than $1 \mathrm{~mm}$ (12). The presence of plaques in the common carotid artery and an increased IMT value correlates well with the results of myocardial perfusion scintigraphic stress testing in patients evaluated for suspected CAD as confirmed by the work of Hallerstam et al with 110 patients (13).

The IMT value also correlates with the incidence of acute myocardial infarction (AMI) $(14,15)$. The IMT itself, but also its irregularity in the examined segment, are predictors of an advanced atherosclerotic process, since a higher level of IMT irregularity can be observed in patients with more advanced CAD (16). Another factor that can be observed in the carotid arteries is the presence of positive remodeling. This term refers to a compensatory reaction of the vessel to plaque progression. By increasing the vessel diameter, the size of the vessel lumen is also increased, thereby compensating for the reduction caused by progression of the atherosclerotic plaque. Kato et al have demonstrated that positive remodeling, an increased IMT value and hypoechogenic and calcified plaques in the carotid arteries can be observed significantly more often in patients with multiple complex lesions of the coronary vessels (17).

Ultrasonographic examination of the carotid arteries allows not only for the assessment of

plaque morphology, but also of plaque echogenity. Less echogenic plaques contain higher amounts of lipids and necrotic matter and are therefore considered high-risk.

Ultrasonographic examination of the carotid arteries is being increasingly employed for the estimation of coronary risk because many patients evaluated during a theoretical examination 24 hours before the occurrence of ACS would have been judged to be at low or intermediate risk solely on the basis of classical risk factors. The ultrasonographic examination of carotid arteries helps in the diagnosis of developed atherosclerotic lesions and may shift the risk profile of a patient from the low- or intermediate-risk to the high-risk group (18).

\section{The use of gene polymorphisms in the prediction of coronary atherosclerosis incidence}

Endothelial dysfunction plays a key role in the pathophysiology of ACS. A procoagulative and proinflammatory phenotype of the endothelium in combination with high vascular tone can facilitate plaque rupture and intraluminal thrombus formation. Nitric oxide (NO) and to a lesser extent carbon monoxide (CO) play a significant role in the regulation of vascular tone. Therefore, the role of pivotal genes involved in the metabolism of these gaseous molecules and also the significance of their selected polymorphisms will be mentioned. These genes encode endothelial nitric oxide synthase (eNOS) and hemoxygenase type 1 (HO1). The product of the first gene is an enzyme that synthesizes NO, a substance with vasodilatory, antithrombotic and antiproliferative properties. Many polymorphisms in the eNOS gene that demonstrate a variable clinical impact have been identified; one of which is the Glu298Asp polymorphism whose significance has been extensively studied. In case of this polymorphism, which is localized in exon 7 of the eNOS gene, the substitution of guanosine $(\mathrm{G})$ for thymidine $(\mathrm{T})$ at position 894 leads to a change in the amino acid sequence of the protein with the substitution of glutamate (Glu) for aspartate (Asp). According to many studies, individuals homozygous for the risk allele (Asp/Asp) are at an increased risk for development of arterial hypertension and coronary atherosclerosis $(19,20)$. The enzyme $\mathrm{HO} 1$ catalyzes the reaction in which the heme is degraded to iron (Fe), $\mathrm{CO}$ and biliverdin, which is subsequently converted to bilirubin. $\mathrm{CO}$ and bilirubin are substances with vasodilatory, antioxidative, angiogenic and anti-inflammatory properties and thus lead to suppression of atherogenesis. The activity of this inducible enzyme is determined by the number of guanosine-thymidine (GT) dinucleotide repeats in the gene promoter. As the number of dinucleotide repeats increases, transcription of the gene, and thus the enzymatic activity, decreases. Many studies have identified a positive correlation between the number of dinucleotide repeats and the development of diseases in which oxidative stress is the underlying pathogenetic mechanism, including $\operatorname{CAD}(21,22)$. Conversely, gene variants with a low number of GT repeats react to oxidative stress with increased transcriptional activity and thus act to protect against the development of atherosclerosis (23).

\section{Proinflammatory cytokines and atherosclerosis}

Vascular cellular adhesive molecules (VCAM-1), intercellular adhesive molecules (ICAM-1) and selectins (E-endothelial, Pplatelet) belong to a group of soluble adhesive molecules, which is a subgroup of the superfamily of immunoglobulins. They are considered to be a marker and mediator of endothelial vasomotor dysfunction and contribute to atherosclerotic plaque progression (24). Their increased expression is believed to correlate with increased cardiovascular risk $(25,26,27)$, and they are thought to participate in all the above-mentioned early processes of atherosclerosis (28). The expression of adhesive molecules on the surface of the endothelium is especially increased in the primarily altered (dysfunctional) endothelium and is influenced by many mechanisms. One of the principal mechanisms with regard to atherogenesis is the regulation of adhesive molecule expression by oxidized LDL particles. Another key mechanism is the regulation by mechanical action of the blood stream, or more precisely by changes in the vessel wall strain (the so-called shear stress). The possibility for noninvasive detection of the atherosclerosis burden and risk stratification by means of adhesive molecule level assessment is currently under cautious debate (29). In particular, ICAM-1 is probably a marker of early atherosclerotic changes. However, according to published data, is rather not an ideal marker for the stratification of ACS risk $(30,31)$. VCAM-1 appears to be more specific and prognostically significant. A significant correlation with plaque progression and cardiovascular changes, along with a significant increase in its levels during the course of an acute coronary syndrome have been described in VCAM-1 (32). 
413-417

In patients with stable angina pectoris who have stable atherosclerotic plaques, the extent of coronary vessel involvement and its progression are better reflected by serum levels of E-selectine (33).

Aside from cytokine levels, the highly-sensitive C-reactive protein (hsCRP) has been proven to be a very useful marker for coronary atherosclerosis. It not only acts as a biomarker, but also plays a role in the development of coronary thrombosis (34). In summary, with regard to the complexity of the pathophysiological processes occurring in atherosclerotic plaques, one cannot assume that a universal biomarker of atherosclerosis capable of monitoring changes in plaque stability and progression and enabling the stratification of high-risk individuals will ever be discovered. In recent years, a more complex approach that combines imaging techniques and soluble marker assessment is being advocated $(35,36,37)$.

\section{Trial evaluation of the significance of noninvasive markers of coronary atherosclerosis}

In spite of all the aforementioned findings regarding the prediction of coronary risk, our knowledge is still insufficient. In a theoretical examination of completely healthy patients 24 hours before the development of an ACS, many individuals would be judged to be at intermediate- or even low-risk. Therefore, we undertook a study that focused on current possibilities for coronary vessel involvement prediction. The goal of the project is to evaluate the possibilities for predicting coronary vessel atherosclerotic involvement and atherosclerotic plaque composition by means of a carotid arteries examination (IMT measurement and plaque echogenity assessment) in combination with an assessment of gene polymorphisms, which play key roles in the metabolism of gaseous molecules that regulate vascular tone, and an assessment of the levels of proinflammatory cytokines. Examination of the coronary vessels was carried out using the following methods: coronary angiography (assessing the extent and severity of atherosclerotic involvement), intravascular ultrasound (assessing the hemodynamic significance of borderline stenoses and plaque volume) and virtual histology (assessing the plaque composition).

Patients in whom CAG was indicated for any reason were enrolled in the trial. In individuals with a borderline stenosis (narrowing of the vessel diameter by $40-70 \%$ as assessed by angiography), the IVUS and VH examinations were carried out to determine the hemodynamic significance. This is a routine examination since IVUS is an appropriate method for determining the hemodynamic significance of lesions (38). If the stenosis was judged to be hemodynamically significant, percutaneous or surgical revascularization was carried out; if not, the patient was treated conservatively. In patients with multiple lesions of the coronary vessels, the IVUS was carried out in the angiographically most significant lesion. In cases of similarly significant stenoses, the one found in the proximal third of one of the main coronary vessels (where plaques most frequently causing ACS are localized) was chosen for IVUS examination. Subsequently, ultrasonographic examination of the carotid arteries was performed, and polymorphisms of the eNOS and $\mathrm{HO} 1$ genes and levels of the following proinflammatory cytokines were assessed: interleukin-6, VCAM-1, ICAM-1, tumor necrosis factor alpha (TNF-alpha), CD 40 ligand and hsCRP. The patients were also classified on the basis of classical atherosclerosis risk factors (smoking, arterial hypertension, diabetes mellitus, total cholesterol, LDL cholesterol, gender and age) according to the Framingham score and the Score risk stratification valid for the Czech population $(39,40)$. Currently, 107 patients for whom CAG and IVUS examinations were indicated have been enrolled in the trial. All patients underwent the aforementioned noninvasive tests. At the moment, the acquired data is being analyzed.

\section{Conclusion}

Based on trials comparing the ultrasonographic findings on the carotid arteries with coronary vessel involvement, it is known that a correlation exists between the atherosclerotic burden in both vessel territories. Likewise, studies have been conducted to evaluate the relationship between the degree of atherosclerosis development and polymorphisms of the genes regulating vascular tone. Finally, studies evaluating the significance of the increased levels of proinflammatory cytokines as markers of coronary atherosclerosis have also been carried out. To our knowledge, no trial to date has evaluated the possibility of coronary vessel atherosclerotic burden prediction by means of a complex examination of all of these risk factors along with "classic" population risks of the development of CAD. We hope that this study will provide valuable information for the noninvasive risk stratification of CAD.

\section{References}

1. Marso S, Frutkin A, Mehta S et al. Intravascular ultrasound measures of coronary atherosclerosis are associated with the Framingham risk score: an analysis from a global IVUS registry. EuroIntervention 2009; 5: 212-218.

2. Hackett D, Verwilghen J, Davies G et al. Coronary stenoses before and after myocardial infarction. Am J Cardiol 1989; 63: 1517-1518.

3. Glagov S, Weisenberg E, Zarins $\mathbf{C K}$ et al. Compensatory enlargement of human atherosclerotic coronary arteries. N Engl J Med 1987; 316: 1371-1375.

4. Loree HM, Kamm RD, Stringfellow RG et al. Effects of fibrous cap thickness on peak circumferential stress in model atherosclerotic vessels. Circ Res 1992; 71: 850-858.

5. Fernadez-Ortiz F, Badimon J, Falk $\mathbf{E}$ et al. Characterization of the relative thrombogenicity of atherosclerotic plaque components: Implications for consequences of plaque rupture. J Am Coll Cardiol 1994; 23: 1562-1569.

6. Yamagishi M, Terashima M, Awano K et al. Morphology of Vulnerable Coronary Plaque: Insights from Follow-up of patients Examined by Intravascular Ultrasound Before an Acute Coronary syndrome. J Am Coll Cardiol 2000; 35: 106-111.

7. Schmermund A, Schwartz RS, Adamzik M et al. Coronary atherosclerosis in unheralded sudden coronary death under age 50: histo-pathologic comparison with "healthy" subjects dying out of hospital. Atherosclerosis 2001; 155: 499-508.

8. Moore MP, Spencer T, Salter DM et al. Characterization of coronary atherosclerotic morphology by spectral analysis of radiofrequency signal: in vitro intravascular ultrasound study with histological and radiological validation. Heart 1998; 79: 459-467. 
9. Garcia-Garcia HM, Mintz GS, Lerman A et al. Tissue characterisation using intravascular radiofrequency data analysis: recommendations for acquisition, analysis, interpretation and reporting. EuroIntervention 2009; 5: 177-189.

10. Stone GW, Maehara A, Lansky AJ et al. PROSPECT: A natural history study of atherosclerosis using multimodality intracoronary imaging to prospectively identify vulnerable plaque. TCT 2009, nepublikovaná data.

11. Nishimura Y, Okamura Y, Fujiwara K et al. Ultrasonographic measurement of intima-media thickness of the carotid artery before coronary artery bypass grafting. Surg Today 2005; 35: 28-30.

12. Kablak-Ziembicka A, Tracz W, Przewlocki T et al. Association of increased carotid intima-media thickness with extent of coronary artery disease. Heart 2004; 90: 1286-1290.

13. Hallerstam S, Larsson PT, Zuber E et al. Carotid atherosclerosis is correlated with extent and severity of coronary artery disease evaluated by myocardial perfusion scintigraphy. Angiology 2004; 55: 281-288.

14. O'Leary DH, Polak JF, Kronmal RA et al. Carotid-artery intima and media thickness as a risk factor for myocardial infarction and stroke in older adults. Cardiovascular Health Study Collaborative Research Group. NEJM 1999; 340: 14-221.

15. Bats ML, Hoes AW, Koudstaal PJ et al. Common carotid intimamedia thickness and risk of stroke and myocardial infarction. The Rotterdam study. Circulation 1997; 96: 1432-1437.

16. Ishizu T, Ishimitsu T, Kamiya $\mathbf{H}$ et al. The correlation of irregularities in carotid arterial intima-media thickness with coronary artery disease. Heart Vessels 2002; 17: 1-6.

17. Kato M, Dote K, Habara S et al. Clinical implication of carotid artery remodeling in acute coronary syndrome. Ultrasonographic assessment of positive remodeling. J Am Coll Cardiol 2003; 42: 1026-1032.

18. Abe Y, Rundek T, Scicca RR et al. Ultrasound assessment of subclinical cardiovascular disease in a community-based multiethnic population and comparison to Framingham score. Am J Cardiol 2006; 98: 1374-1378.

19. Hingorani AD, Liang CF, Fatibene J, Lyon A et al. A common variant of the endothelial nitric oxide synthase (Glu298 $\geq$ Asp) is a major risk factor for coronary artery disease in the UK. Circulation 1999; 100: 1515-1520.

20. Miyamoto Y, Saito Y, Kajiyama N, Yoshimura M et al. Endothelial nitric oxide synthase gene is positively associated with essential hypertension. Hypertension 1998; 32: 3-8.

21. Yamada N, Yamaya M, Okinaga S, Nakayama K. Microsatellite polymorphism in the heme oxygenase-1 gene promoter is associated with susceptibility to emphysema. Am J Hu Genet 2000; 66: 187-195.

22. Kaneda H, Ohno M, Taguchi J, Togo M et al. Heme oxygenase-1 gene promoter polymorphism is associated with coronary artery disease in Japanese patients with coronary risk factors. Arterioscler Thromb Vasc Biol 2002; 22 (1): 1680-1685.

23. Morita T. Hem oxygenase and atherosclerosis. Artherioscler Thromb Vasc Biol 2005; 25: 1786-1795.

24. Hwang SJ, Ballantyne CM, Sharrett AR et al. Circulating adhesion molecules VCAM-1, ICAM-1, and E-selectin in carotid atherosclerosis and incident coronary heart disease cases: the Atherosclerosis Risk In Communities (ARIC) study. Circulation 1997; 96: 4219-4225.
25. Ridker PM, Buring JE, Rifai N. Soluble P-selectin and the risk of future cardiovascular events. Circulation 2001; 103: 491-495.

26. Ridker PM, Hennekens CH, Roitman-Johnson B et al. Plasma concentration of soluble intercellular adhesion molecule 1 and risks of future myocardial infarction in apparently healthy men. Lancet 1998; 351: 88-89.

27. Blankenberg S, Rupprecht HJ, Bickel C et al. Circulating cell adhesion molecules and death in patients with coronary artery disease. Circulation 2001; 104: 1336-1342.

28. Li AC, Glass CK. The macrophage foam cell as a target for therapeutic intervention. Nat Med 2002; 8: 1235-1240.

29. Hamilton AJ, Huang SL, Warnick D et al. Intravascular ultrasound molecular imaging of atheroma components in vivo. J Am Coll Cardiol 2004; 43: 453-460.

30. Parker C, 3rd, Vita JA, Freedman JE. Soluble adhesion molecules and unstable coronary artery disease. Atherosclerosis 2001; 156: 417-424.

31. Paramo JA, Rodriguez JA, Orbe J. Vulnerable plaque versus vulnerable patient: emerging blood biomarkers for risk stratification. Endocr Metab Immune Disord Drug Targets 2007; 7: 195-120.

32. Mulvihill NT, Foley JB, Murphy RT et al. Risk stratification in unstable angina and non-Q wave myocardial infarction using soluble cell adhesion molecules. Heart 2001; 85: 623-627.

33. O'Brien KD, McDonald TO, Chait A et al. Neovascular expression of E-selectin, intercellular adhesion molecule-1, and vascular cell adhesion molecule-1 in human atherosclerosis and their relation to intimal leukocyte content. Circulation 1996; 93: 672-682.

34. Mills R, Deepak LB. The Yin and Yan of arterial inflammation. JACC 2004; 44: 50-52.

35. Madjid M, Zarrabi A, Litovsky $\mathbf{S}$ et al. Finding vulnerable atherosclerotic plaques: is it worth the effort? Arterioscler Thromb Vasc Biol 2004; 24: 1775-1782.

36. Lipinski MJ, Fuster V, Fisher EA et al. Technology insight: targeting of biological molecules for evaluation of high-risk atherosclerotic plaques with magnetic resonance imaging. Nat Clin Pract Cardiovasc Med 2004; 1: 48-55.

37. Nahrendorf M, Jaffer FA, Kelly KA et al. Noninvasive vascular cell adhesion molecule-1 imaging identifies inflammatory activation of cells in atherosclerosis. Circulation 2006; 114: 1504-1511.

38. Takagi A, Tsurumi Y, Suzuki K et al. Clinical Potential of Intravascular Ultrasound for Physiological Assessment of Coronary Stenosis. Circulation 1999; 100: 250-255.

39. Wilson PW, D'Agostino RB, Levy D et al. Prediction of coronary heart disease using risk factors categories. Circulation 1998; 97: 1837-1847.

40. Cífková $R$, Býma $S$, Češka $R$ et al. Prevence kardiovaskulárních onemocnění v dospělém věku. Společné doporučení českých odborných společností. Supplement Cor Vasa 2005; 47: 3-14.

Received May 31, 2011. Accepted September 21, 2011. 